\title{
SSG-Geschenke - Ideen und Empfehlungen Patienteninformation
}

Was schenke ich meinem Kind zur Unterstützung der logopädischen Therapie? Weihnachten naht schließlich! Welchen Ratgeber aus dem Bereich Sprache - Stimme - Gehör kann ich Angehörigen und Patienten empfehlen? Was schenke ich meiner Freundin zur Eröffnung ihrer logopädischen Praxis? Das Team der Schule für Logopädie der MHH hat für Sie - nach Altersgruppen sortiert - Ideen für „SSG-Geschenke“ zusammengestellt.

\section{Bücher \\ Erste Bilder, erste Worte \\ Spanner, Helmut \\ 2011, Ravensburger, Ravensburg \\ $9,95 €$}

ISBN 9783473324378

Was ist ein U-U-Uhu?

Schneider, Peter

2012, Natke, Neuss

$17,80 €$

ISBN 9783936640106

\section{Kommentar}

Großes Bilderklappbuch bereits für

Kinder ab 1 Jahr, wo einzelne Ge-

genstände aus Themenbereichen

wie Garten, Küche oder Kinderzimmer schön dargestellt werden.

Ein stotternder Igel, der unnachgie-

big Antwort auf eine Frage sucht

und sich dabei als sehr mutig, ent-

schlossen und selbstbewusst zeigt.

Der Text ist sehr ansprechend ge-

schrieben und wunderschön bebildert. Für Eltern gibt es am Ende des Buches einige Information rund um das Stottern.

Plitsch, platsch Badespaß

Geisler, Dagmar

2006, Oetinger, Hamburg

$6,20 €$

ISBN 9783789165740

ich auch

Geisler, Dagmar

2003, Oetinger, Hamburg

$5,90 €$

ISBN 9783789165702

\section{Fröschlein fährt Dreirad}

Schneider, Peter

$6,90 €$

ISBN 9783936640144

Alltagsthemen und Gefühle werden in Reimform dargestellt und sind ansprechend illustriert. Sprechfreude und Sprachbewusstsein werden angeregt. Für Kinder von 2 bis 4 Jahren.

Ein sehr ansprechend illustriertes Bilderlesebuch für Vorschulkinder zur Begleitung der Stottertherapie. Der kindgerechte Text ist in Reimform geschrieben und vermittelt Eltern und Kind erstes Wissen über Stottern.

\section{Die Wetterschachtdetektive}

Technische Universität Dortmund

Freier Download des Hörspiels

Sprachförderung für Kinder im Vorund Grundschulalter. Spannende Geschichte mit aktuellem Bezug.

unter

www.wetterschacht-detektive.de

Hier stimmt ja fast gar nichts!

Das große Suchspaß-Wimmel-

buch

Butschkow, Ralf

2012, Bastei Lübbe, Köln, 9,99€

ISBN 9783833903205

Lustiges Wimmelbuch, wo Kinder

Fehler in alltäglichen Situationen

finden können (z.B. ein Blumentopf am Flugzeugfenster). Für Kinder von 4 bis 6 Jahren. Auch als Ausmalbuch erhältlich.
Ohren, Nase, Bauch - das hab'

2011, Natke, Neuss
Bücher

Stottern in der Schule

Thum, Georg

2011, Demosthenes, Köln

$12,50 €$

ISBN 9783921897669

\section{Kommentar}

Auf vielen Seiten erhält der Leser unterlegt mit vielen Fallbeispielen zahlreiche Anregungen, um einen stotternden Schüler in der Schule zu unterstützen. Es werden Möglichkeiten zur Thematisierung des Stotterns, zu Änderungen des Lernumfeldes und zur Reduzierung belastender Anforderungen dargestellt, um dem stotternden Schüler eine gute Teilhabe zu ermöglichen. Gehört in jede Schulbibliothek!

Manchmal stotter' ich eben
de Geus, Eelco

2011, Demosthenes, Köln $9,50 €$

ISBN 9783921897638

\section{Ich glaub' es hakt!}

Infos rund ums Thema Stottern

(Buch + DVD)

Weikert, Kerstin

2011, Demosthenes, Köln

$8,50 €$

ISBN 9783921897614

\section{Hallo, hier ist Felix!}

Klare, Margaret

2008, Demosthenes, Köln

$6,90 €$

ISBN 9783921897522

\section{Dem jungen stotternden Leser (zwi-} schen 7 und 12 Jahren) wird auf ansprechende und kindgerechte Weise Wissenswertes über das Stottern, über die Ursachen und über Gefühle beim Stottern vermittelt. Der Leser wird angeregt, sich eigene positive Eigenschaften und Fähigkeiten bewusst zu machen und das Stottern als erlaubten Teil seiner Persönlichkeit zu akzeptieren. Durch viele Beschreibungen und Erzählungen von anderen betroffenen Kindern und durch Ideen zum Umgang mit Hänseleien erfährt der Leser Hilfestellungen zur eigenen Störungsbewältigung.

Ratgeber für jugendliche Stotternde, der gut geeignet ist, ihnen einen Einblick ins Stottern zu gewähren. Das Buch macht Mut und hat hohen Informationsgehalt. Es ist anschaulich durch Erfahrungsberichte junger Stotterer (s. auch DVD) od. lustige Comics und enthält Verweise auf aktuelle Internetseiten.

Ein spannend, einfühlsam u. realitätsnah geschriebenes Buch über die Gefühlswelt eines jugendlichen Stotternden. Es behandelt die in der Schule, Familie u. im Freundeskreis auftauchenden Schwierigkeiten sowie die durch die Therapie erreichten Veränderungen

\begin{tabular}{ll}
\hline $\begin{array}{l}\text { Aphasie - wenn Sprache } \\
\text { zerbricht }\end{array}$ & $\begin{array}{l}\text { Abhandlung über Aphasie und der } \\
\text { daraus folgenden Lebensverände- } \\
\text { fenen }\end{array}$ \\
$\begin{array}{l}\text { Pullwitt, Erika, Winnecken, Andre- } \\
\text { rungen aus der Sicht einer Angehö- } \\
\text { rigen. Das Buch beleuchtet insbe- } \\
\text { 2012, Schulz-Kirchner, Idstein, } \\
\text { sondere die Situation der mitbetrof- } \\
\text { fenen Angehörigen, die sonst wenig }\end{array}$ & $\begin{array}{l}\text { berücksichtigt werden. } \\
\text { ISBN } 9783824809219\end{array}$ \\
\end{tabular}




\section{Bücher}

Im Lande Gänseklein

Pullwitt, Eriko

2009, Edition Ebersbach, Berlin

$18,00 €$

ISBN 9783869150055

\section{Kommentar}

Die Autorin beschreibt in diesem Roman den mühevollen Alltag mit ihrem sprachbehinderten Ehemann und wie sie lange zurückgestellte Wünsche durch eine neue Begegnung wiederentdeckt. Unprätentiös und mutig erzählt.

Uns traf der Schlag: Wir haben alle daraus gelernt Bergmann, Gregor und Renate 2010, Books on Demand, Norderstedt, 9,90€ ISBN 9783839153208

Stottern im Erwachsenenalter Wendlandt, Wolfgang 2009, Thieme, Stuttgart $49,95 €$

ISBN 9783131290311

Frei Sprechen

Ein Training für Moderatoren und Redner (Buch + CD)

Rossié, Michael

2009, Econ, Berlin

$23,00 €$

ISBN 9783430200103

\section{Singen und Stimme}

Ein Ratgeber für Singende,

Chorleiter(innen), Pädagog(inn)

en und Therapeut(inn)en.

Haupt, Evamarie

2009, Schulz-Kirchner, Idstein,

$8,90 €$

ISBN 978828404344
Die Autoren schildern auf eindrucksvolle Art ihre eigenen Erfahrungen, wie Aphasie ihr Leben verändert hat und wie sie Belastungen, Ängste und Lebenskrisen bewältigen konnten.

Für erwachsene Stotternde, die sich mit dem Gedanken tragen, eine Behandlung zu beginnen. Das Buch vermittelt Grundlagenwissen und Handlungshilfen in Form vieler hilfreicher Arbeitsbögen. Allerdings ist es nicht sehr preisgünstig.

Ein motivierendes Buch für alle, die vor anderen Menschen sprechen. Der Leser wird animiert, die eigenen sprecherischen Ressourcen auf stimmige Art und Weise auszuprobieren. Die einzelnen Kapitel werden mit aussagekräftigen Hörbeispielen untermalt.

Der Ratgeber gibt auf 64 Seiten einen Überblick über die unterschiedlichen Ausprägungen der Gesangsstimme. Dabei werden die stimmlichen Phänomene in unterschiedlichen Lebensaltern und die verschiedenen Stimmtypen berücksichtigt. Tipps für den Umgang mit Problemen beim Singen werden am Ende des Buches beschrieben. Empfehlenswert besonders für Chorsänger.

\section{Spiele Kommentar}

Ratz-Fatz. Aufgepasst und zugefasst

Haba, ca. $22 €$

ASIN BOOOHYIOY

Konzentration, Reaktionsvermögen und Sprachfähigkeit sind gefordert, wenn auf ein Signalwort innerhalb einer vorgelesenen Geschichte, eines Gedichts oder eines Rätsels „ratz-fatz" reagiert werden muss. Ab 3 Jahren.

Tipp: „Ratz- Fatz“-Spiele gibt es in verschiedenen Themen-Varianten und Altersstufen (z.B. Schule, Weihnachten)

Nanu? Merkfähigkeit und Farbkenntnisse

Ravensburger, ca. $8 € \quad$ werden spielerisch gefördert. Ab 4 ASIN B0000TZ51U Jahren.

Wer bin ich? Anhand von Ja/Nein-Fragen muss
Haba, ca. $8 € \quad$ die Person oder der Gegenstand erASIN B000GYWXLO raten werden, der einem zugeordnet wurde. Schönes Familienspiel ! Erfordert etwas „Weltwissen“. Ab 4 Jahren.

Papafant und Elegei Lustiges Tierlegespiele von 4 bis 8 Berliner Spielkarten/ ASS Spiel- Jahren. Für Vorschulkinder auch zur kartenfabrik, ca. $5 €$ ASIN B0018ZKS1M

Die freche Sprechhexe Buntes Spiel zum Reimen und Fin-
Ravensburger, ca. $10 € \quad$ den ähnlich klingender Worte. Für ASIN B000MVZXKE 4-8-jährige, aber besonders geeignet für Vorschulkinder.

Die freche Englisch-Hexe Buntes Spiel mit Begriffen zu verRavensburger, ca. $10 € \quad$ schiedenen Themen des Alltags für ASIN B002ZCXNCS Kinder im Alter von 5-9 Jahren. Für jüngere Kinder auch auf deutsch spielbar, da die englischen Begriffe (vor)gelesen werden müssen.

Mini LÜK Lernsystem Ein Spielkasten zur Lösung und viele Westermann, Lösungsgerät Themen-hefte. Alles ist möglich von ca.14,90 Euro, Spielhefte ab einfacher Zuordnung und Konzent$5,90 €$ ration im Vorschulalter, über Übungshefte für spezielle Laute (Sch, K, S) hin zu Lese- und Rechenlernheften im Schulalter. Die Kinder können ab dem Vorschulalter selbständig ihre Lösungen prüfen.

Birte Ripken für das Team der Schule für Logopädie der MHH, Hannover

überreicht durch Thieme und 